\title{
Water-Repellent Cellulose Fiber Networks with Multifunctional Properties
}

\author{
Ilker S. Bayer, ${ }^{\dagger}$ Despina Fragouli, ${ }^{\dagger}$ Agnese Attanasio, ${ }^{\dagger}$ Barbara Sorce, ${ }^{\dagger}$ Giovanni Bertoni, ${ }^{\ddagger}$ Rosaria Brescia, \\ Riccardo Di Corato, ${ }^{\S}$ Teresa Pellegrino, ${ }^{\ddagger} \S$ Maria Kalyva, ${ }^{\dagger}$ Stefania Sabella, ${ }^{\dagger}$ Pier Paolo Pompa, ${ }^{\dagger}$ \\ Roberto Cingolani, ${ }^{\neq}$and Athanassia Athanassiou ${ }^{\dagger, \neq, \S, *}$ \\ ${ }^{+}$Center for Biomolecular Nanotechnologies @Unile, Istituto Italiano di Tecnologia (IIT), 73010 Lecce, Italy \\ ${ }^{\ddagger}$ Istituto Italiano di Tecnologia (IIT), 16163 Genova, Italy \\ ${ }^{\S}$ National Nanotechnology Laboratory, CNR - Istituto di Nanoscienze, 73100 Lecce, Italy
}

Supporting Information

\begin{abstract}
We demonstrate a simple but highly efficient technique to introduce multifunctional properties to cellulose fiber networks by wetting them with ethyl-cyanoacrylate monomer solutions containing various suspended organic submicrometer particles or inorganic nanoparticles. Solutions can be applied on cellulosic surfaces by simple solution casting techniques or by dip coating, both being suitable for large area applications. Immediately after solvent evaporation, ethyl-cyanoacrylate starts cross-linking around cellulose fibers under ambient conditions because of naturally occurring surface hydroxyl groups and adsorbed moisture, encapsulating them with a hydrophobic polymer shell. Furthermore, by dispersing various functional particles in the monomer solutions, hydrophobic ethyl-cyanoacrylate nanocomposites with desired functionalities can be formed around the cellulose fibers. To exhibit the versatility of the method, cellulose sheets were functionalized with different ethyl-cyanoa-

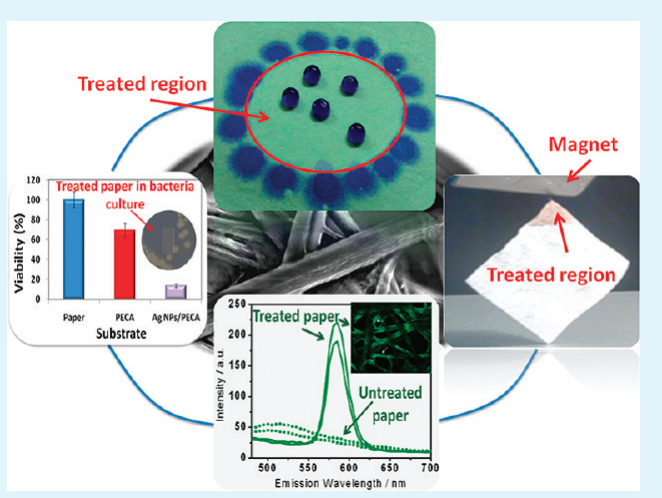
crylate nanocomposite shells comprising submicrometer wax or polytetrafluoroethylene particles for superhydophobicity, $\mathrm{MnFe}_{2} \mathrm{O}_{4}$ nanoparticles for magnetic activity, $\mathrm{CdSe} / \mathrm{ZnS}$ quantum dots for light emission, and silver nanoparticles for antimicrobial activity. Morphological and functional properties of each system have been studied by scanning and transmission electron microscopy, detailed contact angle measurements, light emission spectra and E. coli bacterial growth measurements. A plethora of potential applications can be envisioned for this technique, such as food and industrial packaging, document protection, catalytic cellulosic membranes, textronic (electrofunctional textiles), electromagnetic devices, authentication of valuable documents, and antimicrobial wound healing products to name a few.
\end{abstract}

KEYWORDS: cellulose fibers, cyanoacrylate nanocomposites, antimicrobial, magnetic nanoparticles, quantum dots

\section{INTRODUCTION}

Cellulose fibers with natural origins, such as wood or cotton, are the principal ingredients of many technologically important products, including paper and packaging products, membranes, medical and food products, textiles, etc. Despite their current large scale use, many potential applications of cellulose derivatives are limited by their hygroscopic properties, which can lead to changes in the chemical structure of cellulose, ${ }^{1,2}$ resulting in physical degradation like swelling, loss of mechanical properties, fibers embrittlement, etc. ${ }^{3-5}$ Nonetheless, the application potential of functional cellulosic materials such as electromagnetic or antimicrobial has substantially increased the scientific activity in designing new cellulosic nanomaterials, or functionalizing natural cellulose fibers or sheets. ${ }^{6-20}$ For instance, various recent studies use the cellulose fiber networks, such as paper or cotton sheets, as substrates for the deposition of functional materials for the formation of sensors/actuators or lab on paper devices, ${ }^{10,12-15}$ with the main limitation being the possible degradation due to water absorption, resulting in the loss of any incorporated functionality. On the other hand, interesting studies that demonstrate the use of nanomaterials for the functionalization of cellulose fibers follow mostly complicated and sophisticated preparation methods. ${ }^{6,11,18-20}$ To the best of our knowledge, so far there is not a universal technique that can provide to cellulosic materials such as cellulosic mats, sheets, membranes and paper all the desired functionalities in an inexpensive and single step process, and significantly advance its numerous applications. 8,9 The method we present herein can render cellulosic sheets waterproof with additional functional properties, all in a single step. The technique is simple, low-cost, scalable, and environmentally friendly and can be applied to any cellulosic material composed of cellulose fiber networks. Our top-down

\section{Received: July 7, 2011}

Accepted: September 8, 2011

Published: September 08, 2011 
approach is based on the impregnation of the cellulose fiber networks with solutions of alkylcyanoacrylate (ACA) monomers and submicrometer or nanoscale functional fillers, applied by using drop casting or dip coating, both techniques suitable for scale-up. These monomers are fully biocompatible and in biomedical applications come in direct contact with human skin or open wounds. ${ }^{21}$ The proposed process relies on moisture-initiated polymerization of ACA on the cellulose fibers, because of hydroxyl groups and environmental moisture existence on them, ${ }^{2,22}$ forming a protective polyalkylcyanoacrylate (PACA) layer around each fiber, without changing the structure of entangled fiber network. The PACA cladding around fibers renders them waterproof and at the same time it serves as a matrix to incorporate different kinds of nanomaterials with desired functionalities, such as magnetic actuation, photoluminescence, and antibacterial activity, each one separately or in combination. A number of potential applications of hydrophobic cellulose sheets with multi functional properties can be considered. For instance, in food packaging, hydrophobic cellulose can replace petroleum based polymers that do not biodegrade. Valuable documents can be rendered waterproof during storage against unexpected flooding conditions. Cellulose membranes can be loaded with nanoparticles of specific functionality so that filtration and catalytic conversion can be realized simultaneously. Certain valuable documents can be labeled with this technique and hence they can be traced using special magnetic or spectroscopic techniques. Antimicrobial cellulose sheets can be used as wound dressing and healing materials in medicine.

\section{MATERIALS AND METHODS}

2.1. Materials. Whatman Type- 1 cellulose sheets of $35-40 \mu \mathrm{m}$ thickness were used in all experiments unless otherwise noted. This product is chemically pure and free from silicones or other additives. No chemical or physical pretreatment was applied to the cellulose sheets before the experiments. Ethyl-2-cyanoacrylate (ECA) monomer, yellow Carnauba Wax flakes and submicrometer $(\sim 150 \mathrm{~nm})$ polytetrafluoroethylene (PTFE) particles were purchased from Sigma-Aldrich. Reagent grade solvents such as acetone and toluene were used as received from Sigma-Aldrich. Depending on the application, ECA monomer was dispersed in one of these solvents. Spherical superparamagnetic $\mathrm{MnFe}_{2} \mathrm{O}_{4}$ nanoparticles (NPs) were synthesized using a protocol reported by Zheng et $\mathrm{al}^{23}$ and slightly modified by us. Briefly, $2 \mathrm{mmol}$ of $\mathrm{Fe}(\mathrm{acac})_{3}$, $1.25 \mathrm{mmol}$ of $\mathrm{Mn}(\mathrm{acac})_{2}, 10 \mathrm{mmol}$ of hexadecanediol, $6 \mathrm{mmol}$ of dodecylamine, and $6 \mathrm{mmol}$ of dodecanoic acid were solved in a roundbottom flask with $20 \mathrm{~mL}$ of benzyl ether. The mixture was vigorously mixed under nitrogen for $60 \mathrm{~min}$ at $140^{\circ} \mathrm{C}$, then for $120 \mathrm{~min}$ at $210^{\circ} \mathrm{C}$, and finally for $60 \mathrm{~min}$ at $300^{\circ} \mathrm{C}$. The product was washed four times with a mixture of acetone, isopropanol, and ethanol, and then dispersed in toluene. The average size of the obtained NPs was $9 \pm 1 \mathrm{~nm}$ in diameter. $\mathrm{CdSe}-\mathrm{ZnS}$ core-shell quantum dots (QDs) were synthesized following established synthesis routes. ${ }^{24}$ The concentration was determined using the data of $\mathrm{Yu}$ et $\mathrm{al}^{25}$ for the extinction coefficient. The QDs used for the poly(ethyl-2-cyanoacrylate) (PECA) doping had the emission signal centered at $595 \mathrm{~nm}$ when excited with $405 \mathrm{~nm}$ wavelength, which correspond to an average size of about $6 \pm 1 \mathrm{~nm}$ in diameter. For the entrapping experiments the above-mentioned colloidal nanocrystals were dissolved in toluene ( $25 \mu \mathrm{M}$ for QDs and $19.5 \mu \mathrm{M}$ for $\mathrm{MnFe}_{2} \mathrm{O}_{4}$ ). Silver (Ag) NPs were prepared by laser ablation of an Ag target (diameter 9 $\mathrm{mm}$, thickness $6 \mathrm{~mm}$, purity >99\%) in a solution of $5 \mathrm{wt} \% \mathrm{ECA}$ monomers in acetone. The metal target was placed on the bottom of a glass cuvette filled with $1.5 \mathrm{~mL}$ of the solution. The target was irradiated for $3 \mathrm{~h}$ with the second harmonic $(\lambda=532 \mathrm{~nm})$ of a Nd:YAG laser
(Quanta-Ray GCR-190, Spectra Physics) with pulse duration $\leq 7 \mathrm{~ns}$ and repetition rate of $10 \mathrm{~Hz}$, on a rotation base (speed $4.5 \mathrm{deg} / \mathrm{s}$ ). The laser beam was focused $\sim 1 \mathrm{~mm}$ below the target surface by a quartz lens. The spot diameter was $0.2 \mathrm{~mm}$ and the laser fluence was $18.3 \mathrm{~J} / \mathrm{cm}^{2}$. TEM analysis of the Ag NPs/ECA solution revealed quite monodispersed formed particles with average size around $9 \pm 1 \mathrm{~nm}$. The sizes of all types of nanoparticles were measured by low-resolution TEM on about 100 nanoparticles every time (see the Supporting Information).

2.2. Functionalized Cellulose Fiber Sheets Preparation. To render the sheets hydrophobic, we prepared ECA monomer dispersions (5 wt \%) in acetone and the sheets were either solution cast or dip coated. To increase water repellency of the sheets, we applied wax/ECA or PTFE/ECA composites on the cellulose fibers using solution casting. In a typical procedure, wax/ECA blends in solution were prepared by first melting carnauba wax at $100{ }^{\circ} \mathrm{C}$ on a hot plate in a glass vial. To this was slowly added warm acetone under constant stirring. The solution was then transferred to an ultrasonic processor with a water bath at $40{ }^{\circ} \mathrm{C}$ and mixed for half an hour. Similarly, PTFE/ECA blend solutions were prepared by dispersing PTFE particles in acetone initially using ultrasonic processing for one hour at $25^{\circ} \mathrm{C}$. ECA monomer was then added to the mixture dropwise to form stable ECA/PTFE dispersions in acetone. Dispersions containing wax exceeding 35 wt \% in ECA were not stable. Dispersions containing PTFE particles, however, were stable up to 60 wt $\%$ in ECA. Cellulose sheets treated with various wax/ECA or PTFE/ECA blend solutions were left to dry and polymerize under ambient laboratory conditions overnight. For the preparation of the functionalized magnetic or emitting cellulose sheets, a mother solution of ECA in toluene was prepared with a weight ratio of 5/95. For the magnetic functionalization of cellulose sheets, $1.1 \mathrm{ml}$ of this solution was mixed with $104 \mu \mathrm{l}$ of toluene solution of $\mathrm{MnFe}_{2} \mathrm{O}_{4} \mathrm{NPs}$ corresponding to weight ratio $5 / 95$ of NPs/ECA, and the resulting solution was left under ambient conditions for about two hours, before its application to the cellulose sheets. For the preparation of light emitting cellulose sheets $1.1 \mathrm{ml}$ of the initial ECA solution was mixed with $0.5 \mathrm{ml}$ of toluene and $729 \mu \mathrm{l}$ of toluene solution of $\mathrm{CdSe} / \mathrm{ZnS}$ quantum dots corresponding to a weight ratio 1.6/98.4 of QDs/ECA. Subsequently, pieces of cellulose sheets were dipped in each solution for $15 \mathrm{~s}$ and left to dry under ambient conditions. For the reference samples containing only PECA a piece of sheet was dipped for $15 \mathrm{~s}$ in the initial ECA/toluene solution and left to dry. For the preparation of the antibacterial sheets, pieces of sheets were dipped for $2 \mathrm{~min}$ in the Ag NPs/ECA acetone solution prepared as described by the laser ablation method, and then left to dry initially in acetone atmosphere for $1 \mathrm{~min}$ and subsequently in ambient conditions.

2.3. Characterization. Elemental Analysis. The concentrations of iron, manganese or silver in the NPs solutions were determined by elemental analysis using an ICP-AES spectrometer (iCAP 6500, Thermo). For the analysis, a small aliquot of each sample was dried and subsequently digested in a solution of concentrated $\mathrm{HCl} / \mathrm{HNO}_{3} 3: 1(\mathrm{v} / \mathrm{v})$. Contact angle measurements: Square samples were cut from the treated sheets to measure static contact angle and contact angle hysteresis using a contact angle goniometer (Kruss, Germany). Contact angle hysteresis measurements were done using a home-built inclined stage. Optical microscopy: The microscopy images were acquired by an optical microscope (Carl Zeiss, Axio Scope A1) equipped with a digital camera and image processing software. SEM imaging: SEM images were acquired detecting back scattered electrons on a JEOL JSM-6490LA, working at a pressure of $40 \mathrm{~Pa}$ to reduce sample charging. TEM imaging: Thin crosssectional slices $(100-150 \mathrm{~nm}$ thick) from cellulose fiber networks samples for transmission electron microscopy (TEM) imaging were cut with a Leica UC6 ultramicrotome, after embedding the samples in epoxy resin (Epon 812), and collecting the slices on a carbon coated copper grid. Morphology and size distribution of the laser-ablated Ag NPs were characterized by TEM after depositing $\sim 10 \mu \mathrm{L}$ of diluted solution with acetone on a carbon-coated copper grid, and by letting the 

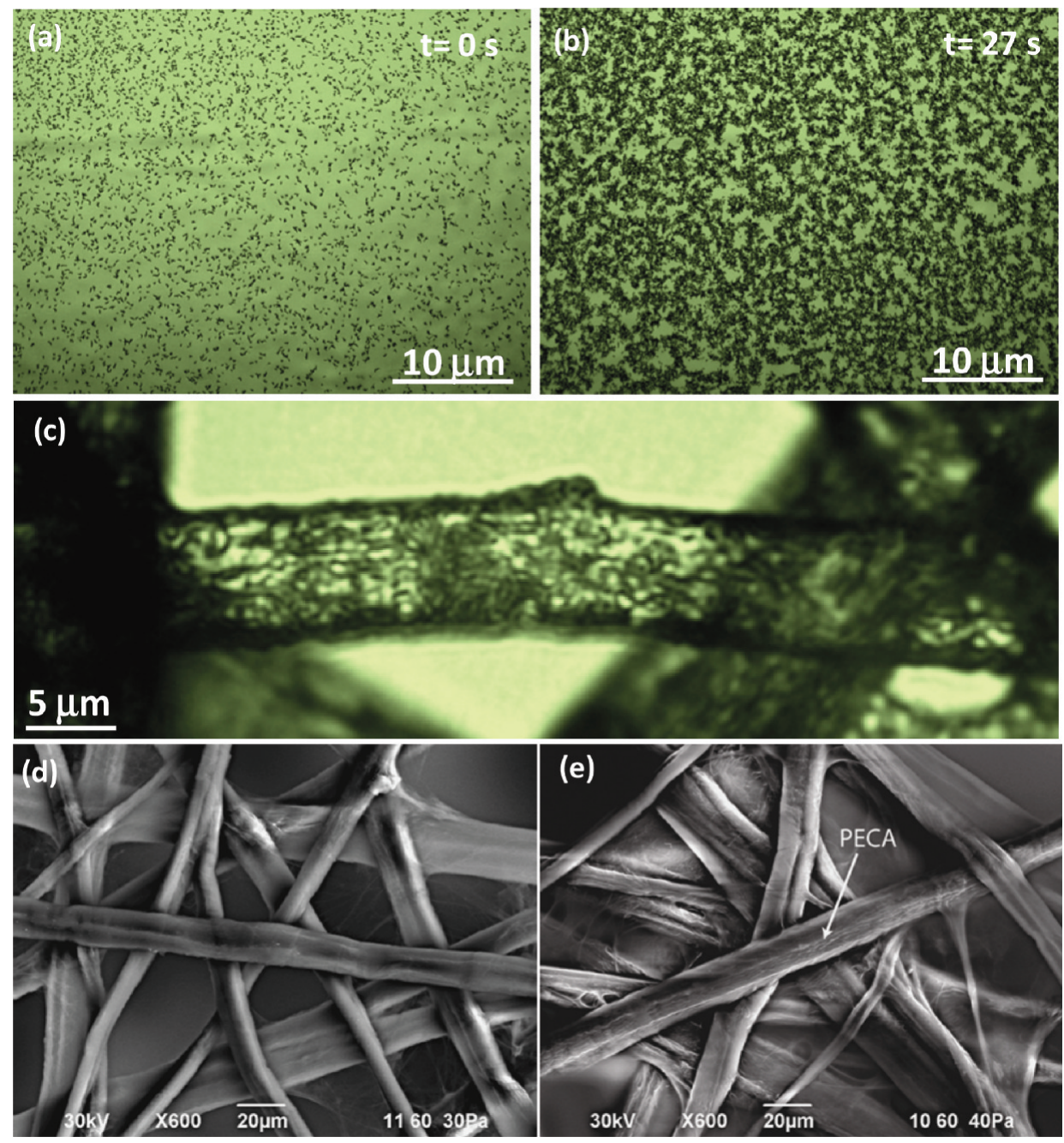

Figure 1. (a, b) Optical microscope images taken at the beginning and at the end of the polymerization of ECA monomers deposited on a glass slide from a 5 wt \% ECA-acetone solution. To accelerate the polymerization, a small beaker of boiling water was placed in the vicinity of the glass slide to increase the moisture levels near the microscope. (c) Optical microscope image of a cellulose fiber wetted by ECA oligomers as they form a network over the fiber surface. SEM images of (d) an untreated cellulosic sheet ( $35 \mu \mathrm{m}$ thickness), and (e) a treated sheet revealing the perfectly preserved fiber network after treatment.

solvent slowly evaporate. All bright-field TEM images were acquired on a JEOL JEM-1011 machine working at $100 \mathrm{kV}$. Confocal imaging: Spatially resolved photoluminescence measurements have been performed on the functionalized emitting sheets by a confocal microscope (Leica TCS-SP5 AOBS). The excitation wavelength $\left(\lambda_{\text {exc }}\right)$ was $405 \mathrm{~nm}$, and the irradiated areas were observed through a $20 \times, 0.70$ NA objective. For spectral analysis, the $\lambda_{\text {exc }}$ was $405 \mathrm{~nm}$, and the emission bandwidth was $20 \mathrm{~nm}$. Analysis of the antibacterial effect: For the antibacterial studies, Lysogeny broth (LB) agar medium was prepared in water. The mixture was taken in a conical flask and autoclaved for $45 \mathrm{~min}$ at $120^{\circ} \mathrm{C}$. The conical flask was kept at room temperature for $2 \mathrm{~h}$ for cooling and then poured in separate Petri dishes. All the cellulose fiber network sheets were sterilized by UV treatment for $10 \mathrm{~min}$. After placing the samples on Petri, a thin layer of LB was further deposited on top. These plates were kept for $1 \mathrm{~h}$ for complete gelation of the agar, and subsequently an E. coli bacterial solution $\left(10^{8} \mathrm{CFU} / \mathrm{mL}\right)$ was uniformly spread over each gel plate in the Petri dishes. The plates were incubated for $24 \mathrm{~h}$ to allow bacterial growth. The bacterial colonies formed in each plate were observed and photographed for comparison (three independent replicates were performed).

\section{RESULTS AND DISCUSSION}

Upon controlled drop casting or dip coating, ECA monomers perfectly wet the cellulose fibers and polymerize. This procedure does not involve sophisticated curing technologies since polymerization occurs rapidly in the presence of humidity, ${ }^{26}$ abundant also at ambient conditions, which is the underlying mechanism in the setting of cyanoacrylate-based adhesives. The evolution of polymerization is clearly demonstrated in the optical microscopic images of Figure 1 ( $a$ and $b$ ), taken immediately after the drop casting onto a glass slide of ECA monomers from an acetone solution, and at the end of their conversion into a polymerized "web", respectively. In the case that the ECA monomer solution is applied onto the cellulose fibers network, the polymerization is catalyzed by the hydroxyl groups of the cellulose $^{3,22}$ and by the humidity of the atmosphere surrounding them. The microscope image in Figure 1c shows the surface of a cellulose fiber after the formation of the polymer shell around it. In fact, the fiber appears covered by the ECA oligomer network demonstrating that each fiber acts as a support for the spreading and the polymerization of the ECA monomers. Therefore, the 


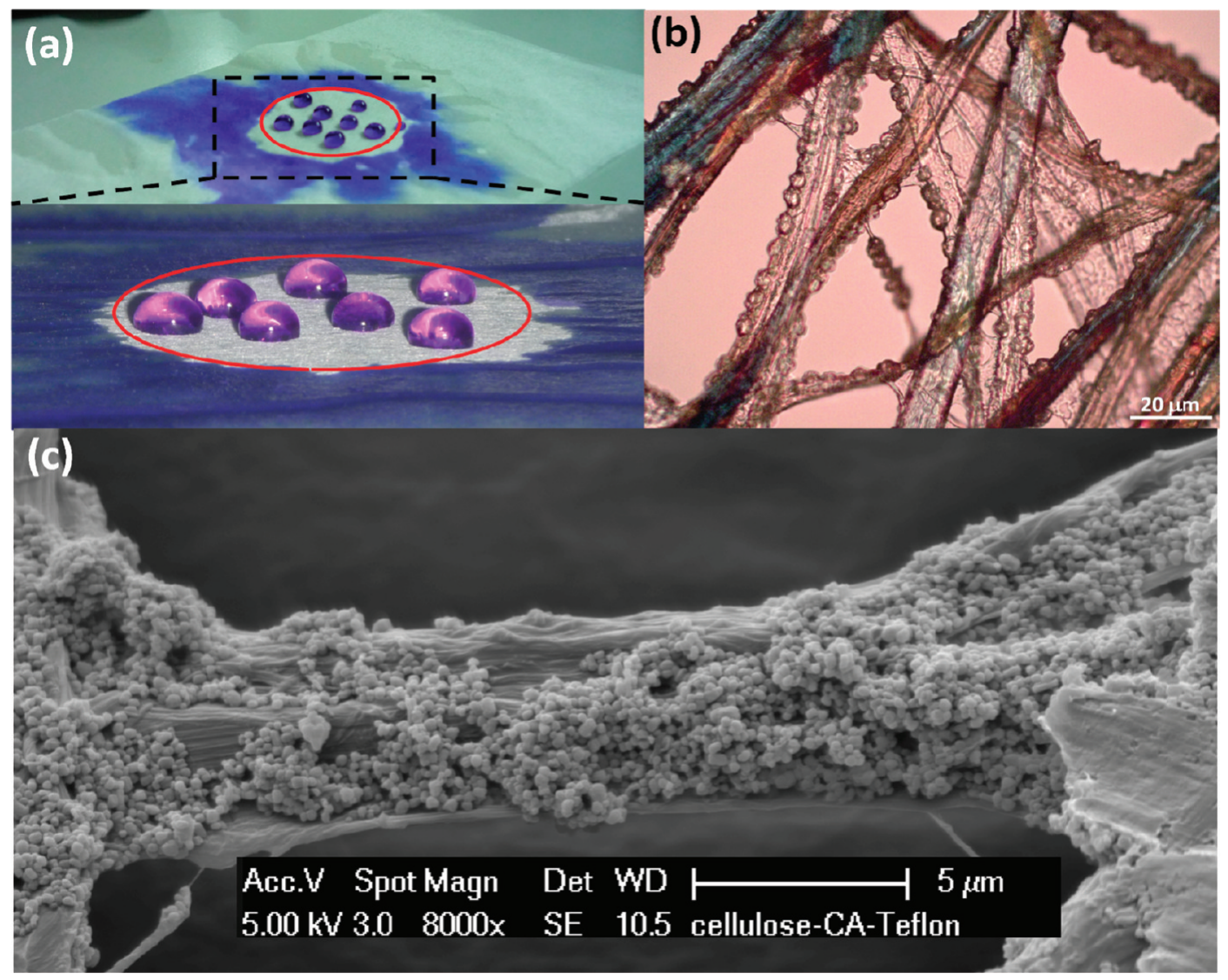

Figure 2. (a) Colored water droplets are laying onto the area of a cellulose sheet treated with PECA (defined by the red line), whereas they are absorbed by its untreated area. (b) Optical microscope image showing surface roughness generation on cellulose fibers by impregnating them with nanocomposite 10.0 wt \% carnauba wax in PECA to render them highly hydrophobic. (c) SEM image showing the surface of a cellulose fiber roughened by submicrometer $(<200 \mathrm{~nm}$ ) PTFE particles mixed with PECA (20.0 wt \% PTFE in PECA) to fabricate super water repellent cellulose sheets.

formed polymer PECA creates a cladding around each individual fiber and not an overall coating onto the cellulose sheet. After the completion of the polymerization, the macroscopic physical characteristics of the sheet such as color and size remain unaltered. Furthermore, scanning electron microscope (SEM) images of the untreated and treated fibers, shown in images $d$ and $\mathrm{e}$ in Figure 1, respectively, reveal that the network of the cellulose fibers seems to be practically unaffected after PECA treatment. It is noteworthy that because of biodegradability of the PACAs, ${ }^{21,27}$ sheets treated in this way can be still recycled.

The PECA shell formed around the cellulose fibers renders them waterproof. Specifically, Figure 2a shows a cellulose sheet (presented in Figure 1d) treated with PECA in its central area. Dyed water drops bead up on the treated part, whereas they completely wet the surrounding nontreated region. It is possible to enhance the water repellent character of the treated fiber network by dispersing plant wax or submicrometer PTFE particles in the initial ECA solution. In this case, the ECA behaves as a vehicle for the delivery and self-assembly of hydrophobic particles on the fibers' surface. After the application of the ECA/wax or ECA/PTFE solution on the cellulose sheets by drop casting, each fiber becomes decorated by the particles, which get bonded to the surface with the PECA, creating a hydrophobic hierarchical surface morphology. Indeed, the optical microscopy and SEM images $\mathrm{b}$ and $\mathrm{c}$ in Figure 2 show the formation of protruding features onto the surface of the fibers, resulting from dispersed wax and PTFE particles, respectively, in the PECA cladding.
The effect of wax or PTFE concentration in PECA on the degree of cellulose sheet hydrophobicity and water hysteresis is shown in Figure 3. In particular, using submicrometer PTFE particles as inclusions in PECA, superhydrophobic $\left(\theta \geq 150^{\circ}\right)$ cellulose sheets can be easily achieved at a minimum of $20 \mathrm{wt} \%$ concentration (Figure 3a). Moreover, as seen in Figure 3b, above this concentration contact angle hysteresis drops below $20^{\circ}$, so that the water droplets can easily roll-off the treated sheet making it self-cleaning. On the other hand, the addition of wax particles in the PECA cladding of the fibers does not render superhydrophobic the treated cellulose sheets, which reach a water contact angle value a bit higher than $130^{\circ}$ (Figure 3a). At the same time, as demonstrated in Figure $3 \mathrm{~b}$, contact angle hysteresis reaches a value of $40^{\circ}$ at wax concentration of $8 \mathrm{wt} \%$ and remains practically stable when this concentration is exceeded. Hence, wax/PECA treated sheets become highly hydrophobic but they do not attain the self-cleaning property. At first this may be seen as a disadvantage; however, applying the same ECA/wax solutions onto ordinary printing papers, we manage to render them water proof, preserving at the same time their printing capacity using ink or laser printers. On the top, because ECA monomer solution wets separately each fiber, no formation of continuous thick films occurs on the surface of the treated sheets, leaving unaltered the macroscopic dimensions of the latter, allowing the treated sheets to be fed normally and printed into any type of printer. Figure $3 \mathrm{c}$ shows the photograph of an ordinary copy paper after solution casting ECA monomers mixed 

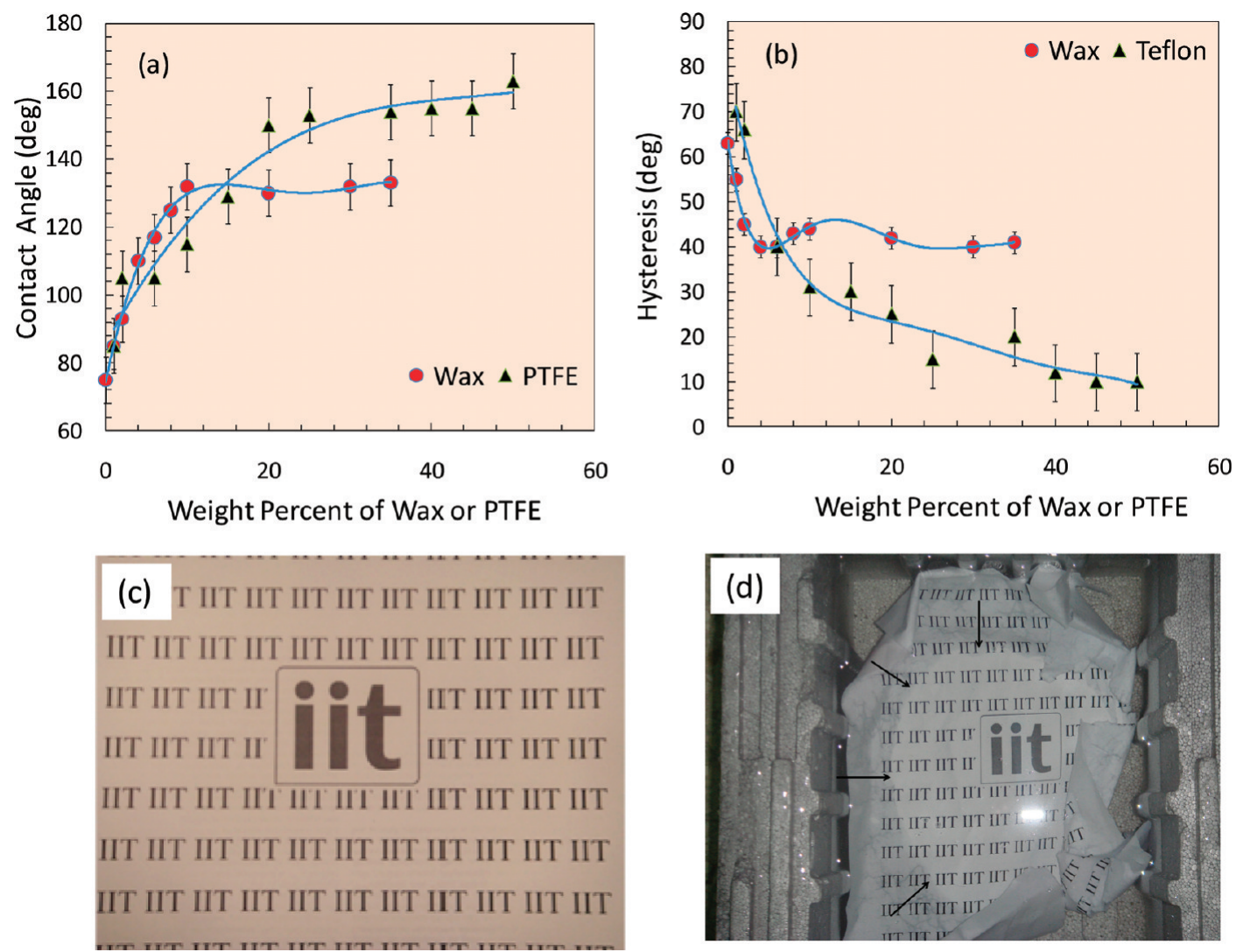

Figure 3. (a) Variation in static water contact angle and (b) contact angle hysteresis for a cellulose sheet impregnated with wax/PECA or PTFE/PECA with respect to particles concentration. Solid lines are drawn to guide the eye. (c) Photograph of an ordinary copy paper locally treated with the wax/ PECA nanocomposite, demonstrating no visible difference in appearance as well as printing quality. (d) Photograph of the paper shown in cimmersed in a water bath. Fifteen minutes after immersion, the untreated paper is disintegrated. The treated region remains intact with the original printing on it.

with wax (75-25 wt \%) only in its central area, and subsequently printing the logo of our institute using a laser printer. No evidence of the treatment can be seen on the paper, demonstrating that its appearance and printing quality remain unaltered. When the paper gets immersed into water bath as seen in Figure $3 \mathrm{~d}$, the central treated area and the print on it remain undamaged, whereas the nontreated area is disintegrated as expected. This biodegradable hydrophobic application can be easily transferred to a large scale application to produce water proof paper with a very low cost.

The presented method has also been exploited in order to tag cellulose fibers with nanoparticles (NPs) having magnetic properties or quantum dots (QDs) having narrow photoluminescence. Magnetic or luminescent sheets are mainly achieved so far by mixing functional particles with suspensions of pulp fibers, resulting in loading the fillers inside the lumens ${ }^{28-30}$ or at the surface of the fibers, ${ }^{31,32}$ by in situ formation of magnetic NPs on cellulose nanofibrils ${ }^{20}$ or fibers, ${ }^{33,34}$ or by wet end addition of particles or dyes. ${ }^{8,18,35}$ The vast majority of those processes are not applicable on cellulosic sheets in their final, postproduction, dry state, as reported herein. Actually, we manage to deliver functional NPs onto the surface of the cellulose fibers by dipping the sheets into solutions containing both nanoparticles and ECA monomers. The nanofillers of choice were superparamagnetic colloidal NPs of manganese ferrite $\left(\mathrm{MnFe}_{2} \mathrm{O}_{4}\right)$ or cadmium selenide/zinc sulphide ( $\mathrm{CdSe} / \mathrm{ZnS})$ QDs prepared with wet chemical methods. During the above-described ECA polymerization around the cellulose fibers, the nanofillers get entrapped into the formed polymeric shell providing magnetic or photoluminescent properties to the cellulose sheets.

Specifically, images a and b in Figure 4 present optical microscope images of cellulose fibers before and after the treatment with the nanocomposite of $5.0 \mathrm{wt} \% \mathrm{MnFe}_{2} \mathrm{O}_{4} \mathrm{NPs}$ in PECA. A comparison between these images shows that the fiber network is still intact after the treatment, with each fiber being covered by the nanocomposite shell. A closer look with cross-sectional transmission electron microscopy (TEM) presented in Figure 4c shows clear evidence of the existence of a composite polymeric layer around each individual fiber where the magnetic NPs are well encapsulated and homogenously dispersed. The hydrophobic character in combination with the nanoroughness of the formed nanocomposite shells of the fibers make the cellulose sheet highly hydrophobic with static water contact angles around $140 \pm 2^{\circ}$. Figure 5a displays a highly absorbing cellulose sheet whose right-bottom corner has been converted into highly hydrophobic after its treatment with $\mathrm{MnFe}_{2} \mathrm{O}_{4} / \mathrm{PECA}$ nanocomposite. But above all, such treatment simultaneously transforms the sheet into magnetically responsive, as demonstrated in the sequence of Figure $5 b-e$, showing how the same piece of cellulose sheet, having only a small corner treated with the magnetic nanocomposite, is easily attracted by a permanent magnet of $0.5 \mathrm{~T}$.

When instead a composite of 1.6 wt \% CdSe/ZnS QDs in PECA is used as the fibers' cladding, the cellulose network becomes highly luminescent. Confocal microscopy measurements, as shown in Figure 6a, reveal that the photoluminescence 

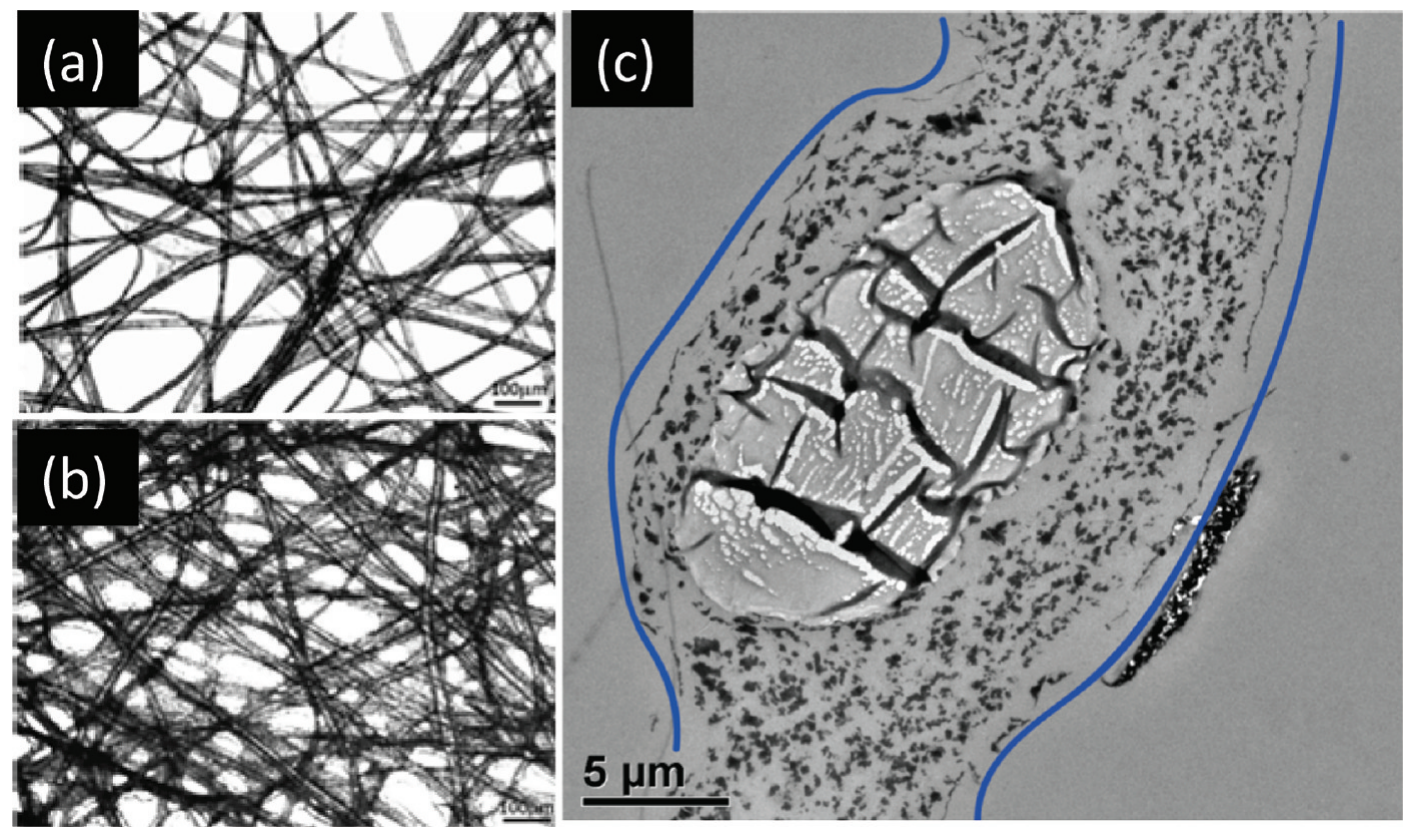

Figure 4. Optical microscopy images of cellulose sheets (a) bare, and (b) treated with nanocomposite of 5 wt $\% \mathrm{MnFe}_{2} \mathrm{O}_{4} \mathrm{NPs}-95$ wt $\%$ PECA. (c) Cross-section TEM image of a cellulose fiber embedded in a shell of the $\mathrm{MnFe}_{2} \mathrm{O}_{4} \mathrm{NPs} / \mathrm{PECA}$ composite.
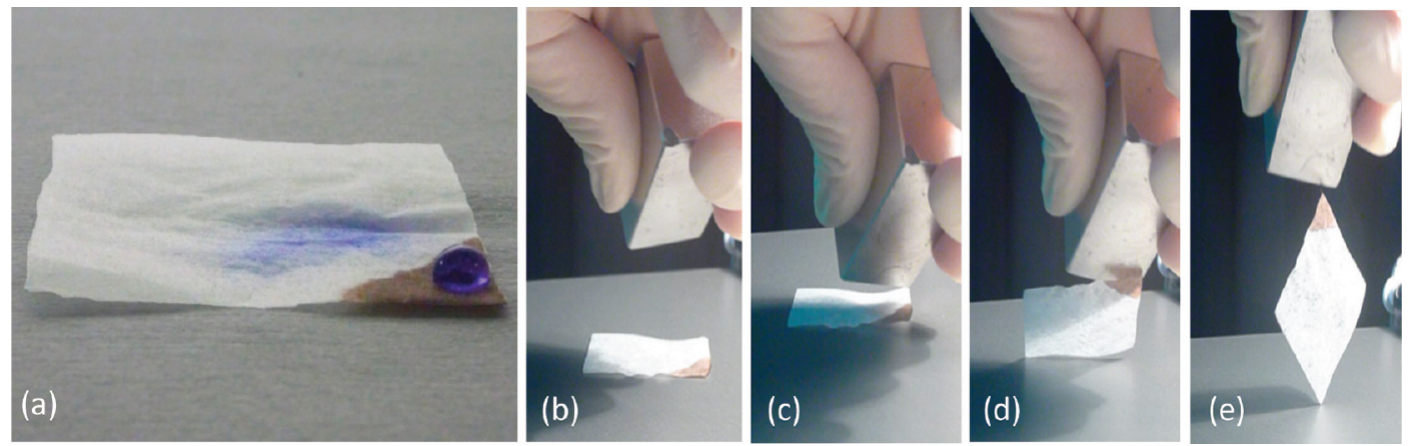

Figure 5. (a) Photo showing the difference in the wetting properties between the corner of a sheet treated with nanocomposite of $5.0 \mathrm{wt} \% \mathrm{MnFe}_{2} \mathrm{O}_{4}$ NPs in PECA (hydrophobic) and the untreated inherently hydrophilic part. (b-e) Magnetic actuation of the treated sheet: A simple magnet attracts the corner of the cellulose sheet treated with the magnetic nanocomposite causing the elevation of the whole piece.

spectra from the treated cellulose fiber network have high intensity and visible emission, typical of the embedded QDs. The respective spectra (Figure 6b) from the untreated or only PECA treated sheet show very broad and low-intensity emission (dashed lines). Whereas, as seen in Figure 6b, the spectra from PECA/QD nanocomposite treated fiber network has a narrow and sharp emission peak centered on ca. $580 \mathrm{~nm}$, characteristic of the used QDs. Note that the technique also allows incorporation of QDs and magnetic NPs simultaneously enhancing multifunctional properties of such cellulosic sheets or paper, i.e., magnetic, luminescent, and hydrophobic. For instance, sheets functionalized in this way can be exploited in order to identify or authenticate the originality of certificates, bank notes, cheques, documents of historic records, etc., through magnetic or optical detection, but they can also be used in various other applications such as microfluidics, actuators, and magneto/optical storage.

Finally, we demonstrate the addition of antibacterial activity to such cellulose fiber networks introducing Ag NPs in PECA. In this case, the NPs were nucleated in situ directly into the ECA monomer solutions, using laser-ablation ${ }^{36}$ of a solid Ag target. The concentration of the Ag NPs was as small as 0.01 wt \% in ECA. This extremely low percentage of Ag NPs is enough to provide the treated sheets with excellent antibacterial properties. To assess the antibacterial effect of Ag NPs/PECA nanocomposite on cellulose fibers, we used Escherichia coli (E. coli) as a model system. The bacterial adhesion and growth were analyzed and compared on untreated cellulosic sheet, on sheets treated only with PECA, and on sheets treated with Ag NPs/PECA nanocomposite, after their immersion in the bacterial culture medium as seen in Figure $7 \mathrm{a}-\mathrm{c}$. Specifically, after $24 \mathrm{~h}$ incubation, a slight decrease of bacterial growth was found on the PECA treated cellulose sheet shown in Figure $7 \mathrm{~b}$, which can be attributed to the known PECA antibacterial properties. ${ }^{37}$ Most importantly, as Figure $7 \mathrm{~d}$ shows, it was observed a large decrease in the number of bacterial colonies in the Ag NPs/PECA-treated sheets compared to the reference untreated sheet. Such antibacterial behavior can be ascribed to the effect of $\mathrm{Ag}+$ ions released by the $\mathrm{Ag}$ NPs dispersed in the PECA cladding of the fibers. Ag+ ions, in 

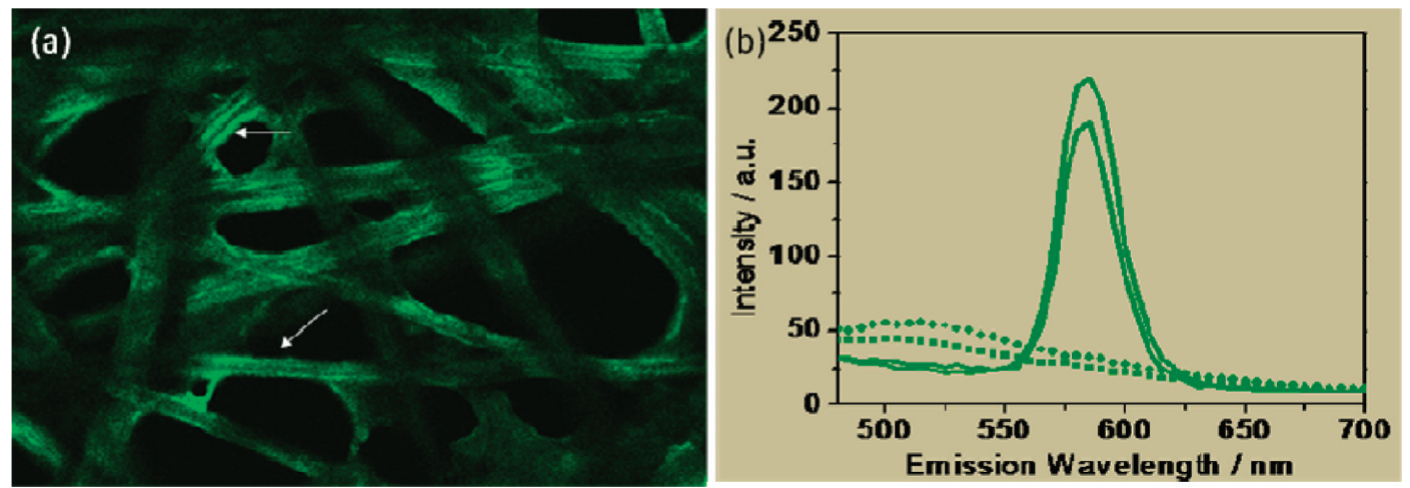

Figure 6. (a) Confocal microscope image showing emission from a cellulose sheet after treatment with the QDs/PECA nanocomposite. (b) Characteristic emission spectra of the QDs identified on the fibers coated by CdSe/ZnS QDs/PECA nanocomposite shown by white arrows in a compared with the low-intensity and broad emission of selected areas from pure or treated only with PECA (dotted lines) cellulosic sheets.
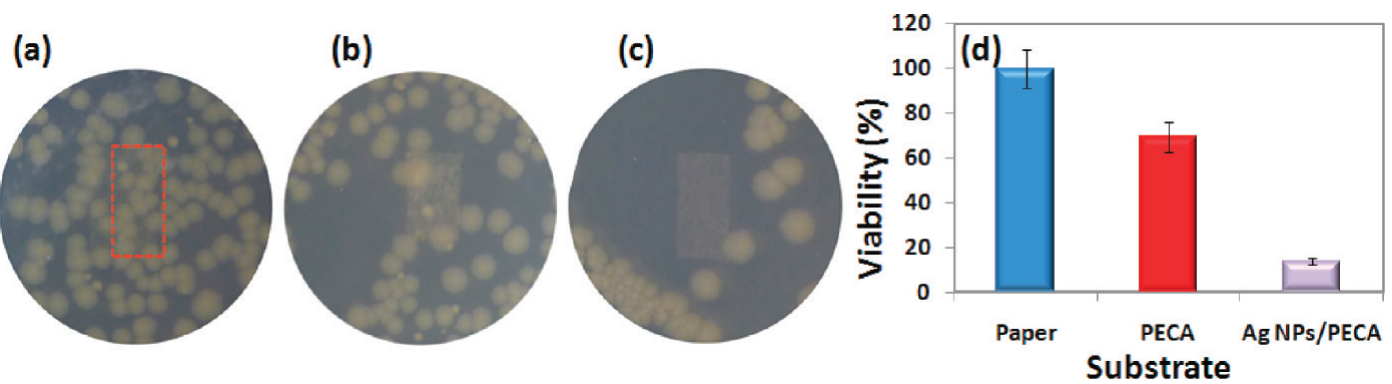

Figure 7. Antibacterial effect after treatment of cellulose sheets with Ag NPs/PECA nanocomposites demonstrated in representative photographs of E. coli bacterial growth (after $24 \mathrm{~h}$ ) on (a) control sample, (b) PECA treated sheet, and (c) Ag NPs/PECA treated sheet. (d) Quantitative analysis of the population of bacteria grown on PECA treated sheet and Ag NPs/PECA treated sheet as compared to control sample (data represent the mean values using 3 independent samples; the error bars indicate the standard deviations).

fact, are well-known to interact with thiol groups of proteins, resulting in the inactivation of respiratory enzymes, and production of reactive oxygen species (ROS) $)^{38}$ that can prevent DNA replication and affect the structure and permeability of cell membranes. ${ }^{39}$

\section{CONCLUSIONS}

In conclusion, we have demonstrated a low-cost, scalable, and fully green process for rendering any kind of fibrous cellulosic material multifunctional. Cellulose fiber networks can be tuned from waterproof to superhydrophobic and selfcleaning, and at the same time they can attain features like magnetic responsivity, specific emissivity, and antibacterial activity. Having in mind the huge variety of cellulose fiber based products, such as packaging materials, paper industry, bank notes, composite construction materials, cotton fabrics, cellulose membranes, etc., the proposed technique of forming functional nanocomposite shells around cellulose fibers can revolutionize their end uses tremendously.

\section{ASSOCIATED CONTENT}

S Supporting Information. TEM images of manganese ferrite, quantum dots, and silver nanoparticles. Absorption and photoluminescence spectra of the quantum dots. This material is available free of charge via the Internet at http://pubs.acs.org.

\section{AUTHOR INFORMATION}

\section{Corresponding Author}

*E-mail: athanassia.athanassiou@iit.it.

\section{REFERENCES}

(1) Topgaard, D.; Soderman, O. Langmuir 2001, 17, 2694.

(2) Hofstetter, K.; Hinterstoisser, B.; Salmen, L. Cellulose 2006, 13, 131.

(3) Alava, M.; Niskanen, K. Rep. Prog. Phys. 2006, 69, 669.

(4) Zou, X.; Gurnagul, N.; Uesaka, T.; Bouchard, J. J. Polym. Degrad. Stab. 1994, 43, 393.

(5) De Spirito, M.; Missori, M.; Papi, M.; Maulucci, G.; Teixeira, J.; Castellano, C.; Arcovito, G. Phys. Rev. E 2008, 77, 041801.

(6) Agarwal, M.; Lvov, Y.; Varahramyan, K. Nanotechnology 2006, 17, 5319.

(7) Khwaldia, K.; Arab-Tehrany, E.; Desobry, S. Compr. Rev. Food Sci. Food Saf. 2010, 9, 82.

(8) Shen, J.; Song, Z.; Qian, X.; Ni, Y. Ind. Eng. Chem. Res. 2011, 50,661 .

(9) Ngo, Y. H.; Li, D.; Simon, G. P.; Garnier, G. Adv. Colloid Interface Sci. 2011, 163, 23.

(10) Tao, H.; Chieffo, L. R.; Brenckle, M. A.; Siebert, S. M.; Liu, M.; Strikwerda, A. C.; Fan, K.; Kaplan, D. L.; Zhang, X.; Averitt, R. D.; Omenetto, F. G. Adv. Mater. 2011, 23, 3197.

(11) Cady, N. C.; Behnke, J. L.; Strickland, A. D. Adv. Funct. Mater. 2011, 21, 2506.

(12) Balu, B.; Berry, A. D.; Hess, D. W.; Breedveld, V. Lab Chip 2009, 9, 3066. 
(13) Hu, L.; Choi, J. W.; Yang, Y.; Jeong, S.; La Mantia, F.; Cui, L.-F.; Cui, Y. Proc. Natl. Acad. Sci. U.S.A. 2009, 106, 21490.

(14) Zhao, W.; van den Berg, A. Lab Chip 2008, 8, 1988.

(15) Lim, W.; Douglas, E. A.; Kim, S.-H.; Norton, D. P.; Pearton, S. J.; Ren, F.; Shen, H.; Chang, W. H. Appl. Phys. Lett. 2009, 94, 072103.

(16) Nogi, M.; Iwamoto, S.; Nakagaito, A. N.; Yano, H. Adv. Mater. 2009, 21, 1595 .

(17) Liu, A.; Walther, A.; Ikkala, O.; Belova, L.; Berglund, L. A. Biomacromolecules 2011, 12, 633.

(18) Niu, T.; Gu, Y.; Huang, J. J. Mater. Chem. 2011, 21, 651.

(19) Shateri Khalil-Abad, M.; Yazdanshenas, M. E. J. Colloid Interface Sci. 2010, 351, 293.

(20) Olsson, R. T.; Azizi Samir, M. A. S.; Salazar-Alvarez, G.; Belova, L.; Ström, V.; Berglund, L. A.; Ikkala, O.; Nogués, J.; Gedde, U. W. Nature Nanotechnol. 2010, 5, 584.

(21) Vauthier, C.; Dubernet, C.; Fattal, E.; Pinto-Alphandary, H.; Couvreur, P. Adv. Drug Deliver. Rev. 2003, 55, 519.

(22) Fras, L.; Laine, J.; Stenius, P.; Stana-Kleinschek, K.; Ribitsch, V.; Dolecek, V. J. Appl. Polym. Sci. 2004, 92, 3186.

(23) Zeng, H.; Rice, P. M.; Wang, S. X.; Sun, S. H. J. Am. Chem. Soc. 2004, 126, 11458.

(24) Dabbousi, B. O.; Rodriguez-Viejo, J.; Mikulec, F. V.; Heine, J. R.; Mattoussi, H.; Ober, R.; Jensen, K. F.; Bawendi, M. G. J. Phys. Chem. B 1997, 101, 9463.

(25) Yu, W. W.; Qu, L. H.; Guo, W. Z.; Peng, X. G. Chem. Mater. 2003, 15, 2854.

(26) Tomlinson, S. K.; Ghita, O. R.; Hooper, R. M.; Evans, K. E. Vib. Spectrosc. 2006, 40, 133.

(27) Han, M. G.; Kim, S.; Liu, S. X. Polym. Degrad. Stab. 2008, 93, 1243.

(28) Chia, C. H.; Zakaria, S.; Ahamd, S.; Abdullah, M.; Jani, S. M. Am. J. Appl. Sci. 2006, 3, 1750.

(29) Zakaria, S.; Ong, B. H.; van de Ven, T. G. M. Colloids Surf. A 2004, 251, 1.

(30) Zakaria, S.; Ong, B. H.; van de Ven, T. G. M. Colloids Surf. A 2004, 251, 31.

(31) Small, A. C.; Johnston, J. H. J. Colloid Interface Sci. 2009, 331, 122.

(32) Small, A. C.; Johnston, J. H. Curr. Appl. Phys. 2008, 8, 512.

(33) Liu, S.; Zhang, L.; Zhou, J.; Wu, R. J. Phys. Chem. C 2008, 112,4538 .

(34) Marchessault, R. H.; Rioux, P.; Raymond, L. Polymer 1992, 33, 4024.

(35) He, Z.; Zhang, H.; Ni, Y.; Zhou, Y. Pulp Pap. 2009, 110, 18.

(36) Kalyva, M.; Bertoni, G.; Milionis, A.; Cingolani, R.; Athanassiou, A. Microsc. Res. Technol. 2010, 73, 937.

(37) Manzano, R. P. D.; Naufal, S. C.; Hida, R. Y.; Guarnieri, L. O. B.; Nishiwaki-Dantas, M. C. Cornea 2006, 25, 350.

(38) Matsumura, Y.; Yoshikata, K.; Kunisaki, S.; Tsuchido, T. Appl. Environ. Microbiol. 2003, 69, 4278.

(39) Feng, Q. L.; Wu, J.; Chen, G. Q.; Cui, F. Z.; Kim, T. N.; Kim, J. O. J. Biomed. Mater. Res. 2000, 52, 662. 